\title{
Microarray-based detection and expression analysis of extracellular matrix proteins in drug-resistant ovarian cancer cell lines
}

\author{
RADOSŁAW JANUCHOWSKI ${ }^{1}$, PIOTR ZAWIERUCHA ${ }^{1,2}$, MARCIN RUCIŃSKI $^{1}$ and MACIEJ ZABEL ${ }^{1,3}$ \\ Departments of ${ }^{1}$ Histology and Embryology, and ${ }^{2}$ Anatomy, Poznań University of Medical Sciences, Poznań 61-781; \\ ${ }^{3}$ Department of Histology and Embryology, Wrocław Medical University, Wrocław 50-368, Poland
}

Received July 14, 2014; Accepted August 18, 2014

DOI: $10.3892 / o r .2014 .3468$

\begin{abstract}
Ovarian cancer is the most lethal gynecological malignancy. Multiple drug resistance (MDR) development leads to resistance of cancer cells to chemotherapy. Microarray methods can provide information regarding new candidate genes that can play a role in resistance to cytostatic drugs. Extracellular matrix (ECM) can influence drug resistance by inhibiting the penetration of the drug into cancer tissue as well as increased apoptosis resistance. In the present study, we report changes in the ECM and related gene expression pattern in methotrexate-, cisplatin-, doxorubicin-, vincristine-, topotecan- and paclitaxel-resistant variants of the W1 ovarian cancer cell line. The resistant variants of the W1 cell line were generated by stepwise selection of cells with an increasing concentration of the indicated drugs. Affymetrix GeneChip ${ }^{\circledR}$ Human Genome U219 Array Strips were used for hybridizations. Independent t-tests were used to determinate the statistical significance of results. Genes whose expression levels were higher than the assumed threshold (upregulated, $>5$-fold and downregulated, $<5$-fold) were visualized using the scatter plot method, selected and listed in the tables. Among the investigated genes, expression of 24 genes increased, expression of 14 genes decreased and expression of three genes increased or decreased depending on the cell line. Among the increased genes, expression of 10 increased very significantly, $>20$-fold. These genes were: ITGB1BP3, COL3A1, COL5A2, COL15A1, TGFBI, DCN, LUM, MATN2, POSTN and EGFL6. The expression of seven genes decreased very significantly: ITGA1, COL1A2, LAMA2, GPC3, KRT23, VIT and HMCN1. The expression pattern of ECM and related genes provided the preliminary view into the role of ECM components in cytostatic drug resistance of cancer cells. The
\end{abstract}

Correspondence to: Dr Radosław Januchowski, Department of Histology and Embryology, Poznań University of Medical Sciences, 6 Swiencickiego Street, Poznań 61-781, Poland

E-mail: rjanuchowski@wp.pl

Key words: multidrug resistance, cDNA microarray, ovarian cancer, extracellular matrix exact role of the investigated genes in drug resistance requires further investigation.

\section{Introduction}

Ovarian cancer is the sixth most common cancer in women (1). A high percentage of mortality results from late diagnosis and low chemotherapy effectiveness. In most cases, ovarian cancer is diagnosed when the disease has progressed to stage III or IV, according to the FIGO classification (2). These patients have a poor prognosis with the current therapies. The first-line treatment includes a combined chemotherapeutic regimen of platinum and taxane (3). The second-line chemotherapy generally includes taxane derivatives as well as cisplatin (Cis), topotecan (Top) and doxorubicin (Dox) $(4,5)$.

The primary reason for low chemotherapy efficacy is resistance of cancer cells to treatment. Several different drugresistance mechanisms have been observed in cancer cells. These mechanisms include: decreased drug accumulation in the cells, change in drug cellular localization, faster inactivation of the drug, faster repair of damage by the drug DNA and cellular membranes, changes in amino acid sequences of target proteins which make them insensitive or less sensitive to the drugs actions as well as changes in regulation of apoptosis. However, the most important and prevalent mechanism of drug resistance is multiple drug resistance (MDR). MDR means the ability of cancer cells to actively remove the cytostatics from the cell via transport proteins. Drug transporters from the ABC family are responsible for these phenomena (6). Among them, P-glycoprotein (P-gp) and breast cancer resistance protein (BCRP) play the main role in MDR.

Sometimes a drug resistance phenotype is observed in spite of a lack in drug resistance gene expression. This suggests that other yet unknown genes may also play a role in cancer drug resistance. Genome wide expression analysis by oligonucleotide microarray may provide a new insight into novel candidate genes that are involved in drug resistance.

Current understanding of drug resistance development in response to cytostatic drugs is based largely on research on drug-sensitive and -resistant pairs of cell lines. By analyzing expression of genes using microarray techniques in six drugresistant ovarian cancer cell lines, we observed changes in 
the expression of many genes related to extracellular matrix (ECM).

ECM consists of ground substance and fibers. These components are a dynamic and interactive system that inform cells about mechanical and biochemical changes in their extracellular environment. The ground substance occupies the space between the cells and fibers and consists of proteoglycans, such as decorin, lumican and keratocan; multi adhesive glycoproteins including fibronectin and laminin; and glycosaminoglycans, such as hyaluronan or keratan sulfate. Among fibers, collagen and elastin fibers can be distinguished. ECM molecules regulate gene expression, cell proliferation, differentiation and migration as well as cancer metastasis (7). In physiological conditions, expression of ECM proteins is restricted to connective tissue. In pathological conditions, ECM expression has been reported in many cancers in vivo (8) and in drug-resistant cancer cell lines (9).

It has been reported that ECM can affect drug resistance in two different ways. First, by preventing the drug penetration in the cancer tissue (10-12). ECM components such as collagen, elastin and polysaccharides (hyaluronan and proteoglycan), may limit drug diffusion (13). Some drugs, such as Cis, are readily distributed within the tumor whereas Dox, methotrexate, vinblastine and paclitaxel bind to cellular macromolecules (14). Second, by interaction of cancer cells with components of ECM and with growth factors. These interactions can affect the apoptosis sensitivity and drug resistance of cancer cells $(15,16)$. This type of drug resistance is designated as cell adhesion-mediated drug resistance (CAM-DR) (17). An example of this is resistance of small cell lung cancer (SCLC) cells to Dox and melphalan, mediated by interaction of $\beta 1$-integrins with ECM (8). Alteration in expression of ECM components, matrix metalloproteinases (MMPs) and other enzymes can lead to ECM remodelling and increased cancer metastasis $(18,19)$.

The present study shows alterations in the gene expression levels of ECM and related proteins in the methotrexate (W1MR), cisplatin (W1CR), doxorubicin (W1DR), vincristine (W1VR), topotecan (W1TR) and paclitaxel (W1PR)-resistant variant of $\mathrm{W} 1$ primary ovarian cancer cell line.

\section{Materials and methods}

Reagents. Methotrexate, Cis, Dox, vincristine, Top and paclitaxel were obtained from Sigma (St. Louis, MO, USA). TRI reagent, RPMI-1640 medium, fetal bovine serum, penicillin, streptomycin, amphotericin B $(25 \mu \mathrm{g} / \mathrm{ml})$ and L-glutamine were also purchased from Sigma. A cell proliferation kit I (MTT) was purchased from Roche Diagnostics GmbH (Mannheim, Germany). Affymetrix GeneChip ${ }^{\circledR}$ 3' IVT Express kit, and Affymetrix GeneChip Human Genome U219 microarrays were both from Affymetrix (Santa Clara, CA, USA).

Cell lines and cell culture. Human ovarian cancer cell line W1 was established from ovarian cancer tissue obtained from an untreated patient. Sublines resistant to Mtx- W1 methotrexate (W1MR), Cis- W1 cisplatin (W1CR), Dox- W1 doxorubicin (W1DR), Vin- W1 vincristine (W1VR), Top- W1 topotecan (W1TR) and Pac- W1 paclitaxel (W1PR) were obtained by exposure of the W1 line to stepwise increasing drug concentra- tions. Final concentration of each drug was 2-fold greater than the concentration in the plasma $2 \mathrm{~h}$ after intravenous administration (20). The cells were 138-, 8-, 10-, 24-, 20- and 641-fold resistant to their selective drugs, respectively, as determined by cell proliferation kit I (MTT). All cell lines were maintained as monolayer in complete medium [RPMI-1640 medium supplemented with $10 \%(\mathrm{v} / \mathrm{v})$ fetal bovine serum, 2 pMl-glutamine, penicillin $(100 \mathrm{U} / \mathrm{ml})$, streptomycin $(100 \mathrm{U} / \mathrm{ml})$ and amphotericin $\mathrm{B}(25 \mu \mathrm{g} / \mathrm{ml})]$ at $37^{\circ} \mathrm{C}$ in a $5 \% \mathrm{CO}_{2}$ atmosphere.

$R N A$ isolation and preparation of microarray and $R Q-P C R$ reactions. RNA isolation from $\mathrm{W} 1$ and all resistant sublines was performed using TRI reagent, according to company protocol. RNA was quantified using spectrophotometry methods by measuring absorbance values at 260 and $280 \mathrm{~nm}$ wave length as well as ratio of $260 / 280 \mathrm{~nm}$ to estimate protein contamination level. Each sample ratio was in (1.8-2.0) interval. RNA degradation level was checked by electrophoresis method using $1 \%$ denaturing agarose gel and was also measured by estimating RIN factor on Bioanalyzer 2100 (Agilent Technologies, Inc., Santa Clara, CA, USA). Estimated RIN values were between 8.5-10 with an average of 9.2. Additionally, each sample was diluted to final work concentration of $100 \mathrm{ng} / \mu \mathrm{l}$. All samples were prepared in triplicate. cDNA for microarray analysis was synthesized in two steps (separate synthesis for first and second strand) using Affymetrix GeneChip ${ }^{\circledR} 3$ 'IVT Express kit and $100 \mathrm{ng} / \mu 1$ of RNA according to Affymetrix GeneAtlas 3' IVT Express kit protocol. Next, in vitro transcription (resulting in population of cRNA), biotin labelling and cRNA fragmentation was also performed using the same protocol.

Microarray hybridization and scanning. Following this procedure, samples were loaded and hybridized with Affymetrix GeneChipHuman Genome U219 microarrays along with control cRNA and oligo B2. The hybridization process was conducted on AccuBlock ${ }^{\mathrm{TM}}$ Digital Dry Bath (Labnet International, Inc. New York, NY, USA) hybridization oven at $45^{\circ} \mathrm{C}$ for $16 \mathrm{~h}$. We then washed and stained microarrays according to the technical protocol using Affymetrix GeneAtlas ${ }^{\mathrm{TM}}$ Fuidics Station and scanned chips with Affymetrix GeneAtlas ${ }^{\mathrm{TM}}$ Imaging Station (both from Affymetrix). Scanned microarrays were saved as *.CEL files on hard disk.

Microarray analysis and gene screening. Quality control (QC) studies were performed using Affymetrix GeneAtlas ${ }^{\mathrm{TM}}$ (Affymetrix) software according to the manufacturer's standards. Secondary quality control studies were evaluated by Partek ${ }^{\circledR}$ Express $^{\text {TM }}$ Software (Partek Inc., St. Louis, MO, USA). Gene screening analysis with QC results as well as statistical algorithms (non-parametric Mann-Whitney U test with $\alpha=0.05$ ) were conducted in the same software. As a result, a table of the most important fold-changes was developed and imported to Pathway Studio ${ }^{\circledR}$ Explore (Ariadne Genomics, Rockville, MD, USA) where pathway studies were performed. Genes with most changes in fold degree between resistant and parental W1 cell lines are listed.

To visualize effects of filtering, we applied gene list to scatter plot with fold-change level of five as a threshold (genes upregulated, >5 fold-change and downregulated, $<-5$ foldchange) (Fig. 1). The same threshold was applied to the gene 


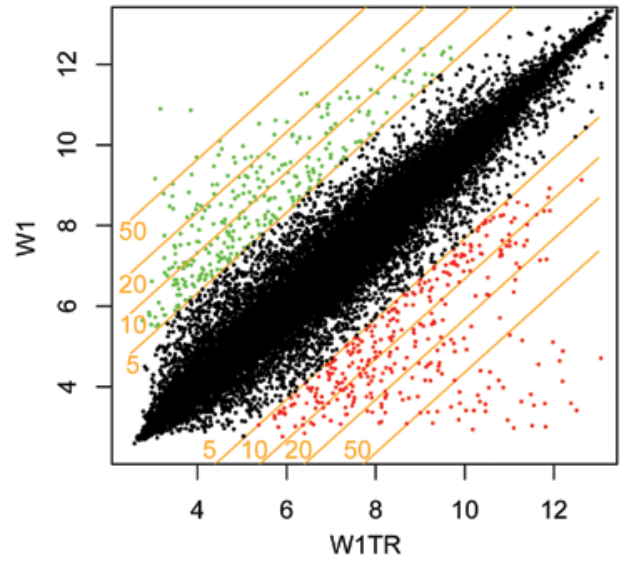

Figure 1. Scatter plot showing the 5-fold upregulated and downregulated genes (the green and red dots, respectively) in the W1TR cell line with respect to the Top-sensitive W1 cell line. The plot filters the genes with fold-change values between 0.2 - and 5-fold (black dots).

table, and in the final step, genes related to ECM were selected and their participation in drug resistant phenotype was evaluated. Scatter plot and gene lists were made using $\mathrm{R}$ language (http://www.r-project.org, version 2.14) with Bioconductor (http://www.bioconductor.org) package installed.

\section{Results}

Gene chip quality assessment. In the present study, we used standard factors such as background to noise signal, internal hybridization controls, internal poly(A) control RNA as well as GAPDH to $\beta$-actin $3^{\prime} / 5^{\prime}$ signal ratios to preliminarily determine the quality of analyzed samples.

Gene expression evaluation and gene expression lists. We analyzed changes in transcription level of ECM-related genes. Analysis of the gene expression in six drug-resistant ovarian cancer cell lines provided new data regarding the response of cancer cells to chemotherapeutics treatment. Tables I and II summarize the changes in the expression of ECM, matrix metallopeptidases and related gene expression levels in drug-resistant sublines with respect to W1 drug sensitive cell line. The genes with statistically significant changes below $\mathrm{p}<0.001$ and within fold-change intervals $(\infty, 5]$ and $[0.2,0)$ which correspond to translation $>5$ and $<-5$, respectively, were considered as genes involved in the drug resistance phenomenon while genes inside interval $(0.2,5)$ were not. Negative values of gene expression were rescaled to positive real numbers so that if $-\mathrm{x}$ is negative expression level than equation $-1 /-\mathrm{x}$ will cast it to positive real numbers set.

Expression of 41 genes encoding ECM proteins, integrin receptors and related genes were altered in drug-resistant cell lines (Tables I and II, Figs. 2 and 3). Twenty-four genes were upregulated in at least one drug-resistant cell line. Fourteen genes were downregulated in at least one drug-resistant cell line and three genes were downregulated or upregulated depending on the cell line. The most variable cell lines were the Pac- and Top-resistant cell lines. In these cell lines we observed changes in expression of 28 and 26 genes, respectively. The most stable cell lines were the Cis- and Mtx-resistant cell lines.

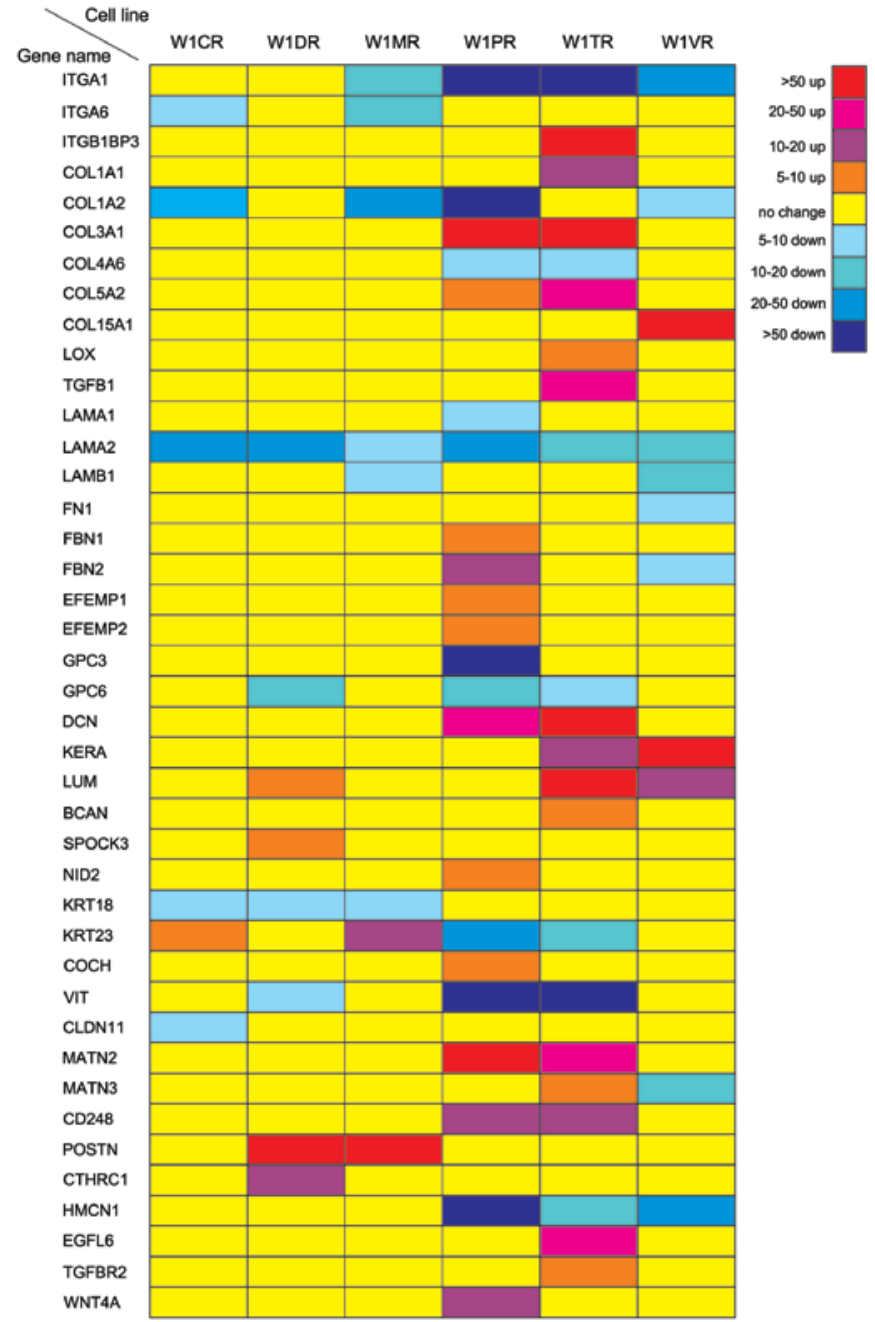

Figure 2. The expression ratios of extracellular matrix genes in the drug-resistant sublines.

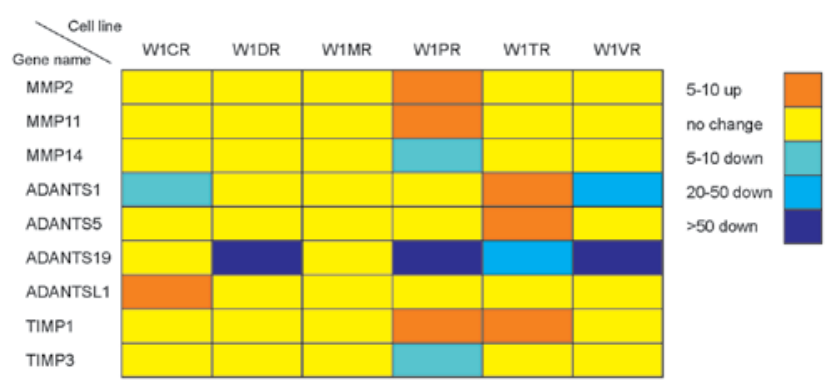

Figure 3 .The expression ratios of matrix metallopeptidases and related genes in the drug-resistant sublines.

In these cell lines, we observed changes in expression of only eight genes.

From these 41 genes, expression of 10 was upregulated very significantly, $>20$-fold. These genes included: ITGB1BP3, COL3A1, COL5A2, COL15A1, TGFBI, DCN, LUM, MATN2, POSTN and EGFL6. Seven genes were very significantly downregulated: ITGA1, COL1A2, LAMA2, GPC3, KRT23, VIT and HMCN1. Changes in expression levels of all genes are summarized in Tables I and II and Figs. 2 and 3. 
Table I. The genes showing the fold-change in extracellular matrix-related proteins in the drug-resistant sublines with respect to the parental W1 cell line.

\begin{tabular}{|c|c|c|c|c|c|c|c|}
\hline \multirow[b]{2}{*}{$\begin{array}{l}\text { Gene } \\
\text { symbol }\end{array}$} & \multirow[b]{2}{*}{$\begin{array}{c}\text { RefSeq } \\
\text { transcript ID }\end{array}$} & \multicolumn{6}{|c|}{ Up-/downregulation } \\
\hline & & $\begin{array}{c}\text { C vs. } \\
\text { W1CR }\end{array}$ & $\begin{array}{l}\text { C vs. } \\
\text { W1DR }\end{array}$ & $\begin{array}{c}\text { C vs. } \\
\text { W1MR }\end{array}$ & $\begin{array}{l}\text { C vs. } \\
\text { W1PR }\end{array}$ & $\begin{array}{l}\text { C vs. } \\
\text { W1TR }\end{array}$ & $\begin{array}{l}\text { C vs. } \\
\text { W1VR }\end{array}$ \\
\hline ITGA1 & NM_181501 & NS & NS & -19.67 & -70.9 & -69.34 & -24.78 \\
\hline ITGA6 & NM_000210 & -6.34 & NS & -19.70 & NS & NS & NS \\
\hline ITGB1BP3 & NM_170678 & NS & NS & NS & NS & 73.56 & NS \\
\hline COL1A1 & NM_000088 & NS & NS & NS & NS & 11.70 & NS \\
\hline COL1A2 & NM_000089 & -28.51 & NS & -29.41 & -64.85 & NS & -5.99 \\
\hline COL3A1 & NM_000090 & NS & NS & NS & 85.11 & $\mathbf{5 4 8 . 7 4}$ & NS \\
\hline COL4A6 & NM_001847 & NS & NS & NS & -6.84 & -5.26 & NS \\
\hline COL5A2 & NM_000393 & NS & NS & NS & 8.95 & 25.09 & NS \\
\hline COL15A1 & NM_001855 & NS & NS & NS & $\mathrm{NS}$ & NS & 66.96 \\
\hline LOX & NM_002317 & NS & NS & NS & NS & 5.72 & NS \\
\hline TGFBI & NM_000358 & NS & NS & NS & NS & 33.78 & NS \\
\hline LAMA1 & NM_005559 & NS & NS & NS & -5.37 & NS & NS \\
\hline LAMA2 & NM_000426 & -27.64 & -38.73 & -6.57 & $\mathbf{- 3 3 . 3 4}$ & -14.22 & -13.23 \\
\hline LAMB1 & NM_002291 & NS & NS & -7.28 & NS & NS & -16.67 \\
\hline FN1 & NM_002026 & NS & NS & NS & NS & NS & -6.14 \\
\hline FBN1 & NM_000138 & NS & NS & NS & 8.87 & NS & NS \\
\hline FBN2 & NM_001999 & NS & NS & NS & 12.50 & NS & -6.13 \\
\hline EFEMP1 & NM_001039348 & NS & NS & NS & 5.10 & NS & NS \\
\hline EFEMP2 & NM_016938 & NS & NS & NS & 5.24 & NS & NS \\
\hline GPC3 & NM_004484 & NS & NS & NS & -63.75 & NS & NS \\
\hline GPC6 & NM_005708 & NS & -14.37 & NS & -14.23 & -8.37 & NS \\
\hline DCN & NM_001920 & NS & NS & NS & 35.32 & 151.11 & NS \\
\hline KERA & NM_007035 & NS & NS & NS & NS & 11.79 & NS \\
\hline LUM & NM_002345 & NS & 6.27 & NS & NS & 137.51 & 11.09 \\
\hline BCAN & NM_021948 & NS & NS & NS & NS & 6.66 & NS \\
\hline SPOCK3 & NM_001040159 & NS & 6.19 & NS & NS & NS & NS \\
\hline NID2 & NM_007361 & NS & NS & NS & 7.20 & NS & NS \\
\hline KRT18 & NM_000224 & -6.08 & -5.35 & -9.12 & NS & NS & NS \\
\hline KRT23 & NM_015515 & 9.71 & NS & 10.61 & -37.96 & -12.63 & NS \\
\hline $\mathrm{COCH}$ & NM_001135058 & NS & NS & NS & 6.77 & NS & NS \\
\hline VIT & NM_053276 & NS & -7.31 & NS & -85.07 & -52.64 & NS \\
\hline CLDN11 & NM_005602 & -6.73 & NS & NS & NS & NS & NS \\
\hline MATN2 & NM_002380 & NS & NS & NS & 60.78 & 29.10 & NS \\
\hline MATN3 & NM_002381 & NS & NS & NS & NS & 7.44 & -11.24 \\
\hline CD248 & NM_020404 & NS & NS & NS & 12.04 & 11.89 & NS \\
\hline POSTN & NM_001135934 & NS & 70.42 & 80.19 & NS & NS & NS \\
\hline CTHRC1 & NM_138455 & NS & 11.11 & NS & NS & NS & NS \\
\hline HMCN1 & NM_031935 & NS & NS & NS & -61.81 & -13.39 & -29.35 \\
\hline EGFL6 & NM_015507 & NS & NS & NS & NS & 20.90 & NS \\
\hline TGFBR2 & NM_001024847 & NS & NS & NS & NS & 5.12 & NS \\
\hline WNT5A & NM_003392 & NS & NS & NS & 18.47 & NS & NS \\
\hline
\end{tabular}

NS, upregulation or downregulation between 5 and -5 , indicative of changes in expression that are not statistically significant.

High similarity between the Pac- and Top-resistant cell lines has been observed. In both cell lines, we observed upregulation and downregulation of six identical genes. Of the six upregulated genes, three were upregulated very significantly in both cell lines: COL3A1, DCN and MATN2. Among the six downregulated genes, three were downregulated very signifi- 
Table II. The genes showing the fold-change in matrix metallopeptidases and related genes in the drug-resistant sublines with respect to the parental $\mathrm{W} 1$ cell line.

\begin{tabular}{llllcccc}
\hline & & \multicolumn{7}{c}{ Up-/downregulation } \\
\cline { 3 - 7 } $\begin{array}{l}\text { Gene } \\
\text { symbol }\end{array}$ & \multicolumn{1}{c}{$\begin{array}{c}\text { RefSeq } \\
\text { transcript ID }\end{array}$} & $\begin{array}{c}\text { C vs. } \\
\text { W1CR }\end{array}$ & $\begin{array}{c}\text { C vs. } \\
\text { W1DR }\end{array}$ & $\begin{array}{c}\text { C vs. } \\
\text { W1MR }\end{array}$ & $\begin{array}{c}\text { C vs. } \\
\text { W1PR }\end{array}$ & $\begin{array}{c}\text { C vs. } \\
\text { W1TR }\end{array}$ & $\begin{array}{r}\text { C vs. } \\
\text { W1VR }\end{array}$ \\
\hline MMP2 & NM_001127891 & NS & NS & NS & $\mathbf{6 . 6 7}$ & NS & NS \\
MMP11 & NM_005940 & NS & NS & NS & $\mathbf{6 . 3 2}$ & NS & NS \\
MMP14 & NM_004995 & NS & NS & NS & $\mathbf{- 7 . 2 5}$ & NS & NS \\
ADAMTS1 & NM_133638 & $\mathbf{- 7 . 7 2}$ & NS & NS & NS & $\mathbf{7 . 8 9}$ & $\mathbf{- 2 4 . 8 5}$ \\
ADAMTS4 & NM_005099 & NS & NS & NS & NS & NS & NS \\
ADAMTS5 & NM_007038 & NS & NS & NS & NS & 5.01 & NS \\
ADAMTS19 & NM_133638 & NS & $\mathbf{- 4 0 . 2 4}$ & NS & $\mathbf{- 9 7 . 8 7}$ & $\mathbf{- 3 8 . 7 7}$ & $\mathbf{- 7 9 . 6 9}$ \\
ADAMTSL1 & NM_001040272 & $\mathbf{5 . 1 3}$ & NS & NS & NS & NS & NS \\
TIMP1 & NM_003254 & NS & NS & NS & $\mathbf{5 . 0 4}$ & $\mathbf{5 . 3 6}$ & NS \\
TIMP3 & NM_000362 & NS & NS & NS & $\mathbf{- 5 . 3 7}$ & NS & NS \\
\hline
\end{tabular}

NS, upregulation or downregulation between 5 and -5 , indicative of changes in expression that are not statistically significant.

cantly in both cell lines: ITGA1, COL1A2 and VIT (Table I and Fig. 2).

Among MMPs, ADAMT and TIMP (Table II and Fig. 3) expression of nine genes was altered in drug-resistant cell lines. Five genes were upregulated, three genes were downregulated and one gene was upregulated or downregulated depending on the cell line. We did not observe any notable changes in upregulated genes. Expression of all genes increased between 5-10-fold. One gene, ADAMTS19, was significantly downregulated $(>20$-fold) in four of six investigated cell lines.

\section{Discussion}

The present study demonstrated a correlation between resistance to cytostatic drugs and expression of genes encoding ECM and related proteins. Genes with a fold-change situated in $(0.2,5)$ interval were considered as not significantly altered in expression and are not taken into consideration.

Expression of drug transporters from the $\mathrm{ABC}$ family is the main reason for the MDR phenotype of cancer in vivo as well as cancer cell lines in vitro. The most important are P-gp and BCRP (21). Expression of these two proteins has also been reported by us in investigated cell lines $(22,23)$. Using western blotting, we confirmed microarray data at the protein level of many other proteins and among them for COL3A1 (data not shown). In all cases, we observed a correlation between microarray results and expression at the protein level.

Previous data indicated that ECM proteins and related molecules may also be involved in cancer drug resistance (17). ECM may increase the drug resistance of a solid tumor by blocking the penetration of therapeutic agents (10-13) or by increasing resistance to apoptosis $(15,16)$. ECM expression has been reported in tumors in vivo as well as in vitro in drug resistant cell lines $(9,24)$. Expression of ECM molecules together with drug transporters from the $\mathrm{ABC}$ family in vitro indicate that ECM components can play a significant role in drug resistance.
Integrins are transmembrane receptors that mediate the attachment between a cell and the ECM. They are involved in cell signalling and the regulation of cell cycle, shape and motility. We observed decreased expression of two integrin genes ITGA1 and ITGA6. This is in contrast to other results, where an increase rather than a decrease in the expression of integrin genes was observed $(9,25,26)$. ITGA1 form with the $\beta 1$ subunit cell-surface receptor for collagen and laminin. Notably, we observed strong downregulation of ITGA1 in cell lines with the highest expression of COL genes. This is in contrast to the study of Varma et al, who observed overexpression of ITGA1 along with COL6A1 in A2780/C10 cell line resistant to oxaliplatin (27). Our result is difficult to explain in the context of studies showing anti-apoptotic effects of cell adhesion to ECM via integrins. In Top-resistant cell line we observed upregulation of the ITGB1BP3 gene. This gene code nicotinamide riboside kinase 2 is also known as muscle integrin-binding protein (MIBP). We did not find any data regarding the role of this protein in cancer drug resistance in the literature. Elucidation of its role in drug resistance requires further investigation.

Upregulation of four collagen genes in investigated cell lines have been observed: COL1A1, COL3A1, COL5A2 and COL15A1. COL3A1 was upregulated in Pac- and Top-resistant cell lines. Expression in Top-resistant cell line increased $>500$-fold. This suggests that in can play a very important role in the resistance to Top. COL1A1 and COL5A2 were also upregulated in Top-resistant cell lines and COL15A1 was upregulated in a Vin-resistant cell line. These results indicate that $\mathrm{COL}$ gene upregulation can be a specific cellular response to Pac, Top and Vin treatment. Similar results were observed by us in other drug-resistant ovarian cancer cell lines resistant to Pac and Top (24). Increased expression of COL genes has also been observed by others in vitro and in vivo. In drug-resistant variants of MCF-7 breast cancer cell line, overexpression of six COL genes was observed (9). In Cis-resistant variant of A2780 ovarian cancer cell line, overexpression of 
COL6A3 and COL11A1 was detected (28). Collagen genes were also upregulated in advanced ovarian cancer (29). These data suggest that collagens indeed play an important role in drug resistance. It appears that COL overexpression can protect cells against cytostatics by two ways. First, by interaction of collagen with cellular receptors. It has been observed that cultivation of Cis-sensitive A2780 ovarian cancer cells in the presence of collagen VI protein led to resistance to Cis in vitro. This effect can result from interaction of collagen with cellular receptors that lead to apoptosis resistance (27). The other possibility is that collagens specifically interact with cytostatic drugs decreasing the amount of drug that can target cells. It has been observed that in tumor resistance to penetration, collagen network is lengthened and this can contribute to drug resistance by preventing the penetration of therapeutic agents (12). Dense and tortuous ECM can be an important barrier for drugs (18). Diffusion rates for larger molecules inversely correlate with level of fibrillar collagen as well as its organization and orientation $(21,30,31)$. We observed considerable expression of fibrillar COL3A1 in Top-resistant cell lines and increased expression in Pac-resistant cell line. Very high expression of COL3A1 in Top-resistant cell lines suggests that it can play a specific role in resistance to this drug. In contrast, expression of COL1A2 and COL4A6 was downregulated in four and two drug-resistant cell lines, respectively, with high downregulation of COL1A2 in W1PR, W1MR and W1CR lines. In the context of other results, it is difficult to elucidate downregulation of these genes in investigated cell lines.

A lysine oxidase (LOX) is important for the crosslinking of collagens and elastin (32). Increase in expression of LOX in W1TR cell lines may be related to expression of many COL genes. Expression of this gene has been reported by us in a Pac-resistant cell line that also expressed many COL genes (24). Increased LOX expression seems to play a critical role in promoting tumor growth and metastasis in many types of cancer, including breast (33), colorectal (34) and lung cancer $(35,36)$. Expression of LOX in W1TR cell line can lead to a more invasive character of this cell line. However, this needs to be confirmed by further study.

Transforming growth factor- $\beta$-induced protein (TGFBI), functions in physiological and pathological conditions including carcinogenesis (37). It can play a tumor suppressor (38) or promoter (39) role depending on the tumor environment. We observed upregulation of TGFBI in Top-resistant cell line. Upregulation of this gene has also been observed by us in other Top-resistant cell lines (24). This indicates that TGFBI can play a role in Top resistance. We did not find any information in the literature regarding the role of TGFBI in Top resistance. TGFBI binds to type I, II and IV collagens. This suggest that it may be important in cell-collagen interactions. It appears that TGFBI expression in Top-resistant cell lines may be related to its interaction with COL1A1 also overexpressed in these cell lines.

Laminins belongs to multiadhesive proteins and play an important role in linking ECM to cell surface. They are trimeric proteins that contain an $\alpha$-, a $\beta$ - and a $\gamma$-chain. The $\alpha$-chain is responsible for cell surface receptor, heparin and heparin sulfate binding. $\beta$ - and $\gamma$-chains are responsible for collagen IV binding. Laminins influence cell differentiation, migration and adhesion (40) and play a role in invasive behavior of tumor cells. In investigated cell lines, we observed decreased expression of three laminin genes with downregulation of LAMA2 in all drug-resistant cell lines. This suggests that downregulation of LAMA2 gene is rather general than specific cell response for cytostatic-induced stress. These results are in contrast to results of Işeri et al, who observed strong upregulation of laminin gene expression in MCF-7 drug resistant sublines (9). It appears that changes in laminin gene expression after cytostatic treatment are cell line-dependent.

FN1 gene encoding fibronectin was slightly downregulated in W1VR cell lines. This is in contrast to other studies that showed increased expression of this gene in drug-resistant cell lines (9). Shibata et al suggested that fibronectin can increase invasiveness of ovarian cancer cells (41).

Fibrillins are microfibrils of ECM and play a role in elastic fiber assembly. FBN1 was upregulated in W1PR cell line. FBN2 was upregulated in W1PR cell line but downregulated in W1VR cell line. Işeri et al also observed upregulation of FBN1 and FBN2 in Pac and other drug-resistant cell lines (9). Thus, it appears that FBN genes can play a role in drug resistance; however, this requires further study.

We observed increased expression of EFEMP1 (also known as fibulin-3) and EFEMP2 (also known as fibulin-4) genes in Pac-resistant cell line. They are members of the fibulin family of ECM glycoproteins. In pancreatic adenocarcinoma, EFEMP1 expression promoted tumor growth in vivo and inhibited apoptosis induced by 5 -fluorouracil, gemcitabine and irinotecan (42). We previously observed very high expression of EFEMP1 in a Dox-resistant cell line (24). Thus, it seems that this gene can indeed play a role in drug resistance. EFEMP2 expression in relation to drug resistance has yet to be fully described.

Glypican (GPC), decorin (DCN), keratocan (KERA), lumican (LUM), brevican (BCAN) and testican encoded by SPOCK3 gene are ECM proteoglycans. It has been reported that some glypicans play a role in cell proliferation and survival. We observed downregulation of two GPC genes, GPC 3 and GPC6, with strong downregulation of GPC 3 in W1PR cell line. This is consistent with observations of Varma et al, who observed GPC3 downregulation in oxaliplatin-resistant ovarian carcinoma cell line A2780/C10 (27). GPC3 expression is frequently silenced in ovarian cancer cell lines and it seems that GPC3 plays a tumor suppressor role in ovarian (43) and lung cancer (44). In both cases, GPC3 expression was silenced by promoter hypermethylation. In contrast, it is upregulated in hepatocellular carcinoma (HCC) and regulates cell proliferation (45). Increased expression of GPC6 has been reported in drug-resistant MCF-7 cell lines (9). We recently observed downregulation as well as upregulation of GPC genes in ovarian cancer cell lines (24). Hence, the role of glypicans in drug resistance remains unclear.

DCN belongs to the small leucine-rich proteoglycan (SLRP) family. It is a component of connective tissue, binds to type I collagen fibrils and plays a role in collagen fibrillogenesis when helps to orient fibers. It also can interact with collagen type I, II, III and IV (46). We observed overexpression of DCN gene in W1TR and W1PR cell lines. High expression in these cell lines can be related to high expression level of type I and III collagen. It has been reported that DCN is a TGF- $\beta 1$ inhibitor and reverses TGF- $\beta 1$-mediated drug resistance (47); 
it also regulates proliferation and migration of A549 lung cancer cells (47) and increases angiogenesis and tumor cell invasiveness in bladder cancer (49). On the one hand, DCN plays an important function in cancer development and metastasis, on the other, it is a potential target in cancer therapy (46).

KERA and LUM are also members of the SLRP family. We observed upregulation of KERA in W1TR cell line and LUM in W1TR as well as in W1VR cell lines. To our knowledge, the relationship between KERA expression and drug resistance has not been described. Increased LUM expression has been reported in Cis-resistant head and neck squamous cell carcinoma cell lines as well as in patients not responding to treatment with Cis-based chemotherapy (50). It has been reported that LUM expression correlates with poor prognosis in advanced colorectal cancer (51). LUM overexpression appears to be a marker of drug resistance, yet its role in this process requires further investigation.

Cytokeratins are intermediate filaments found in the intracytoplasmic cytoskeleton of epithelial tissue. We observed small downregulation of KRT18 in Cis-, Dox- and Mtx-resistant cell lines. Expression of KRT23 was increased in Mtx-resistant cell line but decreased in Pac- and Top-resistant cell lines. To our knowledge, changes in expression of these genes have not been reported in drug-resistant cancer cell lines. Thus, the role of KER gene expression in drug resistance requires further investigation.

Cochlin $(\mathrm{COCH})$ is expressed by fibrocytes in the inner ear and localized to extracellular spaces (52). $\mathrm{COCH}$ expression was slightly increased in Pac-resistant cell line. To our knowledge, to date, its expression in cancer has not been described.

We observed strong downregulation in VIT gene in W1TR and W1PR cell lines. Vitrin was first isolated from the vitreous of the bovine (53). Its role in drug resistance and cancer has not been reported.

Matrilin-2 (MATN2) is a member of the von Willebrand factor A domain containing protein family. It may be involved in the formation of filamentous networks in the ECM, however its specific function has not yet been determined. Its expression has been reported in HCC (54). We observed strong upregulation of MATN2 gene in Pac- and Top-resistant cell lines. This suggests that it may be related to COL gene upregulation in these cell lines. The role of MATN2 in drug resistance has not been described by other researchers. Thus, the exact role of this protein in resistance to cytostatic drugs requires further investigation.

We observed increased expression of CD248, endosialin/ tumor endothelial marker 1 (TEM-1) in Pac- and Top-resistant cell lines. Endosialin is a member of C-type lectin transmembrane receptors which play a role in cell-cell adhesion processes. Its role in cancer neoangiogenesis and tumor growth has been reported $(55,56)$. Thus, it is possible that CD248 expression in vivo can lead to more invasive phenotype of Pac- and Top-resistant cell lines. This, however, requires animal studies.

Periostin (POSTN) is a secreted protein and is a ligand for $\alpha-\mathrm{V} / \beta-3$ and $\alpha-\mathrm{V} / \beta-5$ integrins. It is expressed in epithelial ovarian tumors (29) and plays a role in migration of ovarian epithelial cells $(29,63)$. We observed very high expression level of POSTN in W1DR and W1MR cell line. This is the most highly expressed gene in these cell lines. Xiao et al reported that oxaliplatin or 5-FU-treatment increase POSTN expression in SW480 and HT-29 colon cancer cell lines and lead to chemoresistance through activation of the PI3K/Akt/survivin pathway (58). It is also possible that POSTN induced drug resistance in our investigated cell lines. However, this requires further and more detailed study.

Collagen triple helix repeat containing 1 (CTHRC1) may reduce collagen deposition by inhibition of $\mathrm{Smad} 2 / 3$ activation (59). Its upregulation was reported in gastric cancer as well as in colon cancer cell lines and clinical specimens contributed to cell invasion $(60,61)$. Thus, increased expression of this gene in W1DR cell line can lead to a more invasive character of this cell line.

Hemicentin-1 is a large extracellular member of the immunoglobulin superfamily and is associated with age-related macular degeneration (62). To our knowledge, its role in drug resistance or even cancer has not been described to date. In the present study, we observed its downregulation in W1TR, W1PR and W1VR cell lines. Its role in drug resistance requires further investigation.

We observed EGFL6 overexpression in W1TR cell line. This protein is a member of the epidermal growth factor (EGF) repeat superfamily and is a secreted protein that promotes endothelial cell migration and angiogenesis via ERK activation (63). Its increased expression has been reported in benign meningioma (64), however its role in drug resistance has yet to be described.

Small increase in TGFBR2 encoding TGF, $\beta$ receptor II has been observed in Top-resistant cell line. Overexpression of this protein has also been reported by others in Pac-, Doc- and Dox-resistant variants of MCF-7 breast cancer cell line (65). The authors suggested that this protein stimulated epithelialmesenchymal transition (EMT) in these cell lines. Whether our Top-resistant cell line has characteristics of cells in EMT requires further investigation.

We observed increased expression of WNT5A in W1PR cell line. WNT5A is a secreted protein implicated in oncogenic and several developmental processes. Its expression has been reported in oxaliplatin-resistant ovarian carcinoma cell line A2780/C10 and in pancreatic cancer where it play an important role in drug resistance by increasing resistance to apoptosis $(27,66)$. Thus, its expression in W1PR cell line may be one of the factors that increases its drug resistance.

Matrix metalloproteinases (MMPs) are zinc proteases responsible for ECM degradation. They are a main player in cleaving a number of bioactive molecules and are important in cell proliferation, differentiation and migration. They also play a role in pathological conditions, such as arthritis and cancer metastasis. We observed small changes in MMP expression. MMP2 and MMP11 were increased in W1PR cell line and MMP14 was decreased in this cell line. MMP2 gene encodes an enzyme which degrades type IV collagen. However, we observed downregulation of type IV collagen in W1PR cell line. In this context, increased expression of MMP2 in W1PR cell line is difficult to explain. It has been also reported that MMP2 plays a role in invasion and induces drug resistance of oral squamous cell carcinoma in vitro (67). It is possible that MMP2 plays a similar role in the W1PR cell line. A second MMP overexpressed in W1PR cell line was MMP11. MMP11 is devoid of enzymatic activity against the matrix components 
(68). It has been reported that MMP11 suppresses cancer cell apoptosis and necrosis and promotes tumor development in animal models $(68,69)$. This enzyme is also overexpressed in primary breast cancers, their metastasis and correlates with poor clinical outcome (70). Thus, its expression in the W1PR cell line can play a role in the resistance to apoptosis and may be related to a more invasive character of this cell line. This, however, require further, more detailed study. MMP14 was slightly decreased in the W1PR cell line. It has been described that overexpression of MMP14 in prostate carcinoma induces resistance to gemcitabine by mechanisms involving increases in ERK1/2 phosphorylation (71). The role of this enzyme in resistance to Pac has not been described to date.

ADMST belongs to a disinterring and metalloproteinase with thrombospondin motif family of proteinases. These molecules are involved in various biological events, such as cell adhesion, cell fusion, cell migration, membrane protein shedding and proteolysis. It has been shown that overexpression of ADAMTS-1 promotes metastasis of mammary and lung carcinoma cells (72). We observed increased expression of ADAMTS-1 in a Top-resistant cell line and decreased expression in Cis- and Vin-resistant cell lines. In the study by Işeri et al (9), expression of ADAMST-1 was increased in Vin- and Pac-resistant cell lines. In the context of these results, the role of this enzyme in drug resistance appears to be cell line-dependent.

ADAMTS-5 is known to cleave aggrecan and brevican. Its expression has been reported in human glioblastomas where it cleaves brevican and promotes invasion (73). We observed increased expression of ADAMTS-5 in a Top-resistant cell line. This cell line also overexpresses brevican. Thus, increased expression of ADAMS5 in this cell line may be related to brevican overexpression.

The function of ADAMST-19 remains unclear. The relationship between this gene expression and cancer has also not been described. In the present study, we observed its downregulation in four of six drug-resistant cell lines. Thus, its role in drug resistance and cancer needs to be further investigated.

Tissue inhibitors of metalloproteinases (TIMPs) form inhibitory complexes with MMPs as well as ADAMs and block the ECM degradation. We observed increased expression of TIMP1 in W1PR and W1TR cell lines. In addition to its inhibitory function, TIMP1 can be implicated in the promotion of cell proliferation, inhibition of apoptosis as well as drug resistance. In breast cancer, high levels of TIMP1 have also been associated with a reduced response to chemotherapy $(74,75)$. Thus, its increased expression in investigated cell lines can also be related to chemotherapy resistance.

TIMP3 expression was slightly downregulated in W1PR cell line. This is consistent with results of Işeri et al, who also observed strong downregulation of TIMP3 in MCF-7 cell lines resistant to Doc and Dox (9). Decreased expression of TIMP3 was also reported in ovarian tumors after adjuvant chemotherapy (76). Thus, decreased expression of TIMP3 appears to be an unspecific response of cancer cells to cytostatic drug treatment.

In summary, our results present alteration in expression of many genes encoding ECM-related proteins in six ovarian cancer cell lines resistant to different cytostatic drugs. These results indicate that ECM proteins may also be implicated in drug resistance. Any general response to cytostatic treatment has not been observed. Alterations in gene expression appear to be rather cytostatic-dependent; however, we observed some similarity in gene expression profile between Pac- and Top-resistant cell lines. The importance of the investigated gene expression in drug resistance requires further investigation and should be confirmed in other ovarian cancer cell lines.

\section{Acknowledgements}

This study was funded by grant no. N N401 204139 from the National Science Centre.

\section{References}

1. Garcia M, Jemal A, Ward EM, et al: Global cancer facts \& figures 2007. American Cancer Society, Atlanta, GA, 2007.

2. Kurman RJ (ed): Blaustein's Pathology of the Female Genital Tract. 5th edition. Springer, New York, NY, 2002.

3. Parmar MK, Ledermann JA, Colombo N, du Bois A, Delaloye JF, Kristensen GB, et al: Paclitaxel plus platinum-based chemotherapy versus conventional platinum-based chemotherapy in women with relapsed ovarian cancer: the ICON4/AGO-OVAR-2.2 trial. Lancet 361: 2099-2106, 2003.

4. Pujade-Lauraine E, Wagner U, Aavall-Lundqvist E, et al: Pegylated liposomal doxorubicin and carboplatin compared with paclitaxel and carboplatin for patients with platinum-sensitive ovarian cancer in late relapse. J Clin Oncol 28: 3323-3329, 2010.

5. Sehouli J, Stengel D, Oskay-Oezcelik G, Zeimet AG, Sommer H, Klare $\mathrm{P}$, et al: Non platinum topotecan combinations versus topotecan alone for recurrent ovarian cancer: results of a phase III study of the North-Eastern German Society of Gynecological Oncology Ovarian Cancer Study Group. J Clin Oncol 26: 3176-3182, 2008.

6. Choi $\mathrm{CH}$ : ABC transporters as multidrug resistance mechanisms and the development of chemosensitizers for their reversal. Cancer Cell Int 5: 30, 2005.

7. Stupack DG and Cheresh DA: Get a ligand, get a life: integrins, signaling and cell survival. J Cell Sci 115: 3729-3738, 2002.

8. Sethi T, Rintoul RC, Moore SM, MacKinnon AC, Salter D, Choo C, et al: Extracellular matrix proteins protect small cell lung cancer cells against apoptosis: a mechanism for small cell lung cancer growth and drug resistance in vivo. Nat Med 5: 662-668, 1999 .

9. Işeri OD, Kars MD, Arpaci F and Gündüz U: Gene expression analysis of drug-resistant MCF-7 cells: implications for relation to extracellular matrix proteins. Cancer Chemother Pharmacol 65: 447-455, 2010.

10. Jain RK: The next frontier of molecular medicine: delivery of therapeutics. Nat Med 4: 655-657, 1998

11. Tannock IF, Lee CM, Tunggal JK, Cowan DS and Egorin MJ: Limited penetration of anticancer drugs through tumor tissue: a potential cause of resistance of solid tumors to chemotherapy. Clin Cancer Res 8: 878-884, 2002.

12. Netti PA, Berk DA, Swartz MA, Grodzinsky AJ and Jain RK: Role of extracellular matrix assembly in interstitial transport in solid tumors. Cancer Res 60: 2497-2503, 2000.

13. Jain RK: Transport of molecules in the tumor interstitium: a review. Cancer Res 47: 3039-3051, 1987.

14. Di Paolo A and Bocci G: Drug distribution in tumors: mechanisms, role in drug resistance, and methods for modification. Curr Oncol Rep 9: 109-114, 2007.

15. St Croix B, Flørenes VA, Rak JW, Flanagan M, Bhattacharya N, Slingerland JM and Kerbel RS: Impact of the cyclin-dependent kinase inhibitor p27Kip1 on resistance of tumor cells to anticancer agents. Nat Med 2: 1204-1210, 1996.

16. St Croix B and Kerbel RS: Cell adhesion and drug resistance in cancer. Curr Opin Oncol 9: 549-556, 1997.

17. Dalton WS: The tumor microenvironment as a determinant of drug response and resistance. Drug Resist Updat 2: 285-288, 1999.

18. Chauhan VP, Stylianopoulos T, Boucher Y and Jain R: Delivery of molecular and nanoscale medicine to tumors: transport barriers and strategies. Annu Rev Chem Biomol Eng 2: 281-298, 2011. 
19. Ramanujan S, Pluen A, McKee TD, Brown EB, Boucher Y and Jain RK: Diffusion and convection in collagen gels: implications for transport in the tumor interstitium. Biophys J 83: 1650-1660, 2002.

20. Dietel M, Bals U, Schaefer B, Herzig I, Arps H and Zabel M: In vitro prediction of cytostatic drug resistance in primary cell cultures of solid malignant tumours. Eur J Cancer 29A: 416-420, 1993.

21. Leonard GD, Fojo T and Bates SE: The role of ABC transporters in clinical practice. Oncologist 8: 411-424, 2003

22. Januchowski R, Zawierucha P, Andrzejewska M, Ruciński M and Zabel M: Microarray-based detection and expression analysis of $\mathrm{ABC}$ and SLC transporters in drug-resistant ovarian cancer cell lines. Biomed Pharmacother 67: 240-245, 2013.

23. Januchowski R, Wojtowicz K, Sujka-Kordowska $P$, Andrzejewska M and Zabel M: MDR gene expression analysis of six drug-resistant ovarian cancer cell lines. Biomed Res Int 2013 241763, 2013.

24. Januchowski R, Zawierucha P, Ruciński M, Nowicki M and Maciej Zabel: Extracellular matrix proteins expression profiling in chemoresistant variants of the A2780 ovarian cancer cell line. Biomed Res Int 2014: 365867, 2014.

25. Liang Y, Meleady P, Cleary I, McDonnell S, Connolly L and Clynes M: Selection with melphalan or paclitaxel (Taxol) yields variants with different patterns of multidrug resistance, integrin expression and in vitro invasiveness. Eur J Cancer 37: 1041-1052, 2001.

26. Correia AL and Bissell MJ: The tumor microenvironment is a dominant force in multidrug resistance. Drug Resist Updat 15: $39-49,2012$.

27. Varma RR, Hector SM, Clark K, Greco WR, Hawthorn L and Pendyala L: Gene expression profiling of a clonal isolate of oxaliplatin-resistant ovarian carcinoma cell line A2780/C10. Oncol Rep 14: 925-932, 2005.

28. Sherman-Baust CA, Weeraratna AT, Rangel LB, Pizer ES Cho KR, Schwartz DR, Shock T and Morin PJ: Remodeling of the extracellular matrix through overexpression of collagen VI contributes to cisplatin resistance in ovarian cancer cells. Cancer Cell 3: 377-386, 2003

29. Ismail RS, Baldwin RL, Fang J, Browning D, Karlan BY, Gasson JC and Chang DD: Differential gene expression between normal and tumor-derived ovarian epithelial cells. Cancer Res 60: 6744-6749, 2000.

30. Brown E, McKee T, diTomaso E, Pluen A, Seed B, et al: Dynamic imaging of collagen and its modulation in tumors in vivo using second-harmonic generation. Nat Med 9: 796-800, 2003.

31. Stylianopoulos T, Diop-Frimpong B, Munn LL and Jain RK Diffusion anisotropy in collagen gels and tumors: the effect of fiber network orientation. Biophys J 99: 3119-3128, 2010.

32. Csiszar K: Lysyl oxidases: a novel multifunctional amine oxidase family. Prog Nucleic Acid Res Mol Biol 70: 1-32, 2001.

33. El-Haibi CP, Bell GW, Zhang J, Collmann AY, Wood D, Scherber CM, Csizmadia E, Mariani O, Zhu C, Campagne A, Toner M, Bhatia SN, Irimia D, Vincent-Salomon A and Karnoub AE: Critical role for lysyl oxidase in mesenchymal stem cell-driven breast cancer malignancy. Proc Natl Acad Sci USA 109: 17460-17465, 2012.

34. Baker AM, Cox TR, Bird D, Lang G, Murray GI, Sun XF, Southall SM, Wilson JR and Erler JT: The role of lysyl oxidase in SRC-dependent proliferation and metastasis of colorectal cancer. J Natl Cancer Inst 103: 407-424, 2011.

35. Shi W, Yang B, Li X, Sun S, Wang L and Jiao S: The effect of lysyl oxidase polymorphism on susceptibility and prognosis of nonsmall cell lung cancer. Tumour Biol 33: 2379-2383, 2012.

36. Wilgus ML, Borczuk AC, Stoopler M, Ginsburg M, Gorenstein L, Sonett JR and Powell CA: Lysyl oxidase: a lung adenocarcinoma biomarker of invasion and survival. Cancer 117: 2186-2191, 2011.

37. Ween MP, Oehler MK and Ricciardelli C: Transforming growth factor-beta-induced protein (TGFBI)/(3ig-H3): a matrix protein with dual functions in ovarian cancer. Int J Mol Sci 13 10461-10477, 2012.

38. Li B, Wen G, Zhao Y, Tong $\mathrm{J}$ and Hei TK: The role of TGFBI in mesothelioma and breast cancer: association with tumor suppression. BMC Cancer 12: 239, 2012.

39. Ma C, Rong Y, Radiloff DR, Datto MB, Centeno B, Bao S, Cheng W, Lin F, Jiang S, Yeatman TJ and Wang XF: Extracellular matrix protein $\beta$ ig-h3/TGFBI promotes metastasis of colon cancer by enhancing cell extravasation. Genes Dev 22: 308-321, 2008 .
40. Timpl R, Rohde H, Robey PG, Rennard SI, Foidart JM and Martin GR: Laminin - a glycoprotein from basement membranes. J Biol Chem 254: 9933-9937, 1979.

41. Shibata K, Kikkawa F, Nawa A, Suganuma N and Hamaguchi M: Fibronectin secretion from human peritoneal tissue induces $M_{\mathrm{r}}$ 92,000 type IV collagenase expression and invasion in ovarian cancer cell lines. Cancer Res 57: 5416-5420, 1997.

42. Seeliger H, Camaj P, Ischenko I, Kleespies A, De Toni EN, Thieme SE, Blum H, Assmann G, Jauch KW and Bruns CJ: EFEMPI expression promotes in vivo tumor growth in human pancreatic adenocarcinoma. Mol Cancer Res 7: 189-198, 2009.

43. Lin H, Huber R, Schlessinger D and Morin PJ: Frequent silencing of the GPC3 gene in ovarian cancer cell lines. Cancer Res 59: 807-810, 1999 .

44. Kim H, Xu GL, Borczuk AC, Busch S, Filmus J, Capurro M, Brody JS, Lange J, D'Armiento JM, Rothman PB and Powell CA: The heparan sulfate proteoglycan GPC3 is a potential lung tumor suppressor. Am J Respir Cell Mol Biol 29: 694-701, 2003.

45. Miao HL, Pan ZJ, Lei CJ, Wen JY, Li MY, Liu ZK, Qiu ZD, Lin MZ, Chen NP and Chen M: Knockdown of GPC3 inhibits the proliferation of Huh7 hepatocellular carcinoma cells through down-regulation of YAP. J Cell Biochem 114: 625-631, 2013.

46. Sofeu Feugaing DD, Götte M and Viola M: More than matrix: the multifaceted role of decorin in cancer. Eur J Cell Biol 92: 1-11, 2013.

47. Teicher BA1, Ikebe M, Ara G, Keyes SR and Herbst RS: Transforming growth factor- $\beta 1$ overexpression produces drug resistance in vivo: reversal by decorin. In Vivo 11: 463-472, 1997.

48. Liang S, Xu JF, Cao WJ, Li HP and Hu CP: Human decorin regulates proliferation and migration of human lung cancer A549 cells. Chin Med J 126: 4736-4741, 2013

49. El Behi M, Krumeich S, Lodillinsky C, Kamoun A, Tibaldi L, Sugano G, De Reynies A, Chapeaublanc E, Laplanche A, Lebret T, Allory Y, Radvanyi F, Lantz O, Eiján AM, BernardPierrot I and Théry C: An essential role for decorin in bladder cancer invasiveness. EMBO Mol Med 5: 1835-1851, 2013.

50. Yamano Y, Uzawa K, Saito K, Nakashima D, Kasamatsu A, Koike $\mathrm{H}$, et al: Identification of cisplatin-resistance related genes in head and neck squamous cell carcinoma. Int J Cancer 126: 437-449, 2010.

51. Seya T, Tanaka N, Shinji S, Yokoi K, Koizumi M, Teranishi N, Yamashita K, Tajiri T, Ishiwata T and Naito Z: Lumican expression in advanced colorectal cancer with nodal metastasis correlates with poor prognosis. Oncol Rep 16: 1225-1230, 2006.

52. Robertson NG, Resendes BL, Lin JS, Lee C, Aster JC, Adams JC and Morton CC: Inner ear localization of mRNA and protein products of $C O C H$, mutated in the sensorineural deafness and vestibular disorder, DFNA9. Hum Mol Genet 10: 2493-2500, 2001.

53. Mayne R, Ren ZX, Liu J, Cook T, Carson M and Narayana S: VIT-1: the second member of a new branch of the von Willebrand factor A domain superfamily. Biochem Soc Trans 27: 832-835, 1999.

54. Szabó E, Korpos E, Batmunkh E, Lotz G, Holczbauer A Kovalszky I, Deák F, Kiss I, Schaff Z and Kiss A: Expression of matrilin-2 in liver cirrhosis and hepatocellular carcinoma. Pathol Oncol Res 14: 15-22, 2008

55. Rupp C, Dolznig H, Puri C, Sommergruber W, Kerjaschki D, Rettig WJ and Garin-Chesa P: Mouse endosialin, a C-type lectin-like cell surface receptor: expression during embryonic development and induction in experimental cancer neoangiogenesis. Cancer Immun 6: 10, 2006

56. Valdez Y, Maia M and Conway EM: CD248: reviewing its role in health and disease. Curr Drug Targets 13: 432-439, 2012.

57. Gillan L, Matei D, Fishman DA, Gerbin CS, Karlan BY and Chang DD: Periostin secreted by epithelial ovarian carcinoma is a ligand for $\alpha_{\mathrm{V}} \beta_{3}$ and $\alpha_{\mathrm{V}} \beta_{5}$ integrins and promotes cell motility. Cancer Res 62: 5358-5364, 2002

58. Xiao ZM, Wang XY and Wang AM: Periostin induces chemoresistance in colon cancer cells through activation of the PI3K/Akt/ survivin pathway. Biotechnol Appl Biochem: Dec 24, 2013 (Epub ahead of print). doi: 10.1002/bab.1193.

59. LeClair R and Lindner V: The role of collagen triple helix repeat containing 1 in injured arteries, collagen expression, and transforming growth factor $\beta$ signaling. Trends Cardiovasc Med 17: 202-205, 2007.

60. Wang P, Wang YC, Chen XY, Shen ZY, Cao H, Zhang YJ, Yu J, Zhu JD, Lu YY and Fang JY: CTHRCl is upregulated by promoter demethylation and transforming growth factor- $\beta 1$ and may be associated with metastasis in human gastric cancer. Cancer Sci 103: 1327-1333, 2012. 
61. Kim HC, Kim YS, Oh HW, Kim K, Oh SS, Kim JT, Kim BY, Lee SJ, Choe YK, Kim DH, Kim SH, Chae SW, Kim KD and Lee HG: Collagen triple helix repeat containing 1 (CTHRC1) acts via ERK-dependent induction of MMP9 to promote invasion of colorectal cancer cells. Oncotarget 5: 519-529, 2014.

62. Thompson CL, Klein BE, Klein R, Xu Z, Capriotti J, Joshi T, Leontiev D, Lee KE, Elston RC and Iyengar SK: Complement factor $\mathrm{H}$ and hemicentin-1 in age-related macular degeneration and renal phenotypes. Hum Mol Genet 16: 2135-2148, 2007.

63. Chim SM, Qin A, Tickner J, Pavlos N, Davey T, Wang H, Guo Y, Zheng $\mathrm{MH}$ and $\mathrm{Xu}$ J: EGFL6 promotes endothelial cell migration and angiogenesis through the activation of extracellular signalregulated kinase. J Biol Chem 286: 22035-22046, 2011.

64. Wang X, Gong Y, Wang D, Xie Q, Zheng M, Zhou Y, Li Q, Yang Z, Tang H, Li Y, Hu R, Chen X and Mao Y: Analysis of gene expression profiling in meningioma: deregulated signaling pathways associated with meningioma and EGFL6 overexpression in benign meningioma tissue and serum. PLoS One 7: e52707, 2012.

65. Işeri OD, Kars MD, Arpaci F, Atalay C, Pak I and Gündüz U: Drug resistant MCF-7 cells exhibit epithelial-mesenchymal transition gene expression pattern. Biomed Pharmacother 65: 40-45, 2011.

66. Griesmann H, Ripka S, Pralle M, Ellenrieder V, Baumgart S, Buchholz M, Pilarsky C, Aust D, Gress TM and Michl P. WNT5A-NFAT signaling mediates resistance to apoptosis in pancreatic cancer. Neoplasia 15: 11-22, 2013.

67. Lu L, Xue X, Lan J, Gao Y, Xiong Z, Zhang H, Jiang W, Song W and Zhi Q: MicroRNA-29a upregulates MMP2 in oral squamous cell carcinoma to promote cancer invasion and anti-apoptosis. Biomed Pharmacother 68: 13-19, 2014.

68. Boulay A, Masson R, Chenard MP, et al: High cancer cell death in syngeneic tumors developed in host mice deficient for the stromelysin-3 matrix metalloproteinase. Cancer Res 61: 2189-2193, 2001.
69. Wu E, Mari BP, Wang F, Anderson IC, Sunday ME and Shipp MA: Stromelysin-3 suppresses tumor cell apoptosis in a murine model. J Cell Biochem 82: 549-555, 2001.

70. Noël A, Boulay A, Kebers F, et al: Demonstration in vivo that stromelysin-3 functions through its proteolytic activity. Oncogene 19: 1605-1612, 2000.

71. Dangi-Garimella S, Krantz SB, Barron MR, Shields MA, Heiferman MJ, Grippo PJ, Bentrem DJ and Munshi HG: Threedimensional collagen I promotes gemcitabine resistance in pancreatic cancer through MT1-MMP-mediated expression of HMGA2. Cancer Res 71: 1019-1028, 2011.

72. Liu YJ, Xu Y and Yu Q: Full-length ADAMTS-1 and the ADAMTS-1 fragments display pro- and antimetastatic activity, respectively. Oncogene 25: 2452-2467, 2006.

73. Nakada M, Miyamori H, Kita D, et al: Human glioblastomas overexpress ADAMTS-5 that degrades brevican. Acta Neuropathol 110: 239-246, 2005.

74. Schrohl AS, Meijer-van Gelder ME, Holten-Andersen MN, Christensen IJ, Look MP, Mouridsen HT, et al: Primary tumor levels of tissue inhibitor of metalloproteinases-1 are predictive of resistance to chemotherapy in patients with metastatic breast cancer. Clin Cancer Res 12: 7054-7058, 2006.

75. Klintman M, Ørnbjerg Würtz S, Christensen IJ, Braemer Hertel P, Fernö M, Malmberg M, et al: Association between tumor tissue TIMP-1 levels and objective response to first-line chemotherapy in metastatic breast cancer. Breast Cancer Res Treat 121: 365-371, 2010.

76. L'Espérance S, Popa I, Bachvarova M, Plante M, Patten N, Wu L, Têtu B and Bachvarov D: Gene expression profiling of paired ovarian tumors obtained prior to and following adjuvant chemotherapy: Molecular signatures of chemoresistant tumors. Int J Oncol 29: 5-24, 2006. 